\title{
The Pathology of Hereditary Breast Cancer
}

Sunil R. Lakhani

BCLC, Department of Histopathology, Royal Free and University College Medical School, University College London, Rockefeller Building, University Street, London WC1E 6JJ, $U K$

\section{INTRODUCTION}

A small proportion of breast cancers (5\%) is due to a hereditary predisposition which is transmitted as a highly penetrant autosomal dominant trait. The BRCA1 gene is located on chromosome 17q21 and encodes for a protein of 1863 amino acids. Mutations in BRCA1 gene are associated with a risk of breast cancer of approximately $80 \%$ and a risk of ovarian cancer of approximately $40 \%$ by the age of 70 . The BRCA2 gene is located on chromosome 13q12q13 and encodes for a protein of 3418 amino acids. Mutations in BRCA2 gene are associated with a risk of breast cancer similar to, or higher than that for BRCA1, but a lower risk of ovarian cancer. BRCA2 gene mutations however confer higher risk of male breast cancer. Mutations in BRCA1 and BRCA2 genes together account for approximately $80 \%$ of families with four or more cases of breast cancer diagnosed under the age of 60 years. Certain morphological types including medullary carcinoma, tubular carcinoma, lobular carcinoma in situ, and invasive lobular carcinoma have all been reported to be more commonly associated with a positive family history of breast cancer than other subtypes. It has been difficult to interpret the data in some studies due to the small number of samples, the differing criteria as to what constitutes a positive family history and the evolving criteria for the diagnosis and classification of breast cancer.

\section{METHODS}

The histological findings in familial cases were compared with those in controls unselected for a family history of the disease. There were 440 familial cases of which 118 (27\%) were in the BRCA1 group, $78(18 \%)$ in the BRCA2 group and 82 non-BRCA1/2 cases. The control group comprised 548 breast cancers without a known family history. This represents the largest analysis of both BRCA1 and BRCA2 cases in the literature. Familial cancer specimens were received from participating groups within the UK, USA, Ireland, France, Germany, Iceland, Switzerland, and the Netherlands. Seven pathologists, all of whom had experience of breast pathology carried out the review. The pathologists were unaware as to whether the slides were from the familial or sporadic set. Each of the slides was reported independently by two pathologists.

\section{RESULTS}

(a) Histological Type: More carcinomas were recorded as medullary or atypical medullary in a BRCA1 group (14\%) compared to controls $(2 \%)$. $(\mathrm{p}<0.0001)$. No excess of medullary or atypical medullary carcinoma was seen in the BRCA2 group. An association with lobular carcinoma was found in the non-BRCA1/2 group (odds ratio 8.2)

(b) Histological Grade: The overall grade for BRCA1 and BRCA2 breast cancers was significantly higher than that of the control population. The higher grade of the BRCA1 tumours was a result of higher score of all three parameters of grade. In contrast the higher grade of BRCA2 tumours was only due to higher score for tubule formation. Non-BRCA1/2 tumours 
were lower grade than the BRCA1 and BRCA2 sets.

(c) Multifactorial analysis: The only factors found to be significant for BRCA1 were total mitotic count, continuous pushing margins, and lymphocytic infiltrate. All other features, including the diagnosis of medullary and atypical medullary carcinoma were no longer significant in this analysis. A similar analysis performed for the BRCA2 mutation carriers showed that the only significant features were tubule score, and continuous pushing margins

\section{CLINICAL IMPLICATIONS}

(a) Prognosis: Grade is an independent prognostic indicator and is inversely related to outcome. Hence, patients with mutations in BRCA1 should have a worse prognosis. Paradoxically, familial and medullary carcinomas are said to have a better prognosis than ordinary ductal carcinomas-NST. A recent study suggests that disease-free interval and survival are not different from patients with sporadic breast cancers. In contrast, Foulkes et al., have shown that the worse prognosis predicted by the higher grade is indeed correct. They have demonstrated a poorer survival in node negative patients with BRCA1 mutations compared to the control group.

(b) Genetic Testing: Patients without a family history who develop breast cancers between the age 25-29 years have a risk of approximately $7 \%$ for carrying mutations in the BRCA1 gene. On the assumption that the odds ratio from the analysis of the morphological features is independent of age, a patient under 30 years who has all three features (high mitotic count, continuous pushing margins and prominent lymphocytic infiltrate) would have a risk of $45 \%$ of harbouring a mutation in BRCA1. In the absence of these features the risk would be $2 \%$. Hence the use of morphological features in addition to the clinical data may enhance the counselling of patients, who are likely to harbour mutations in this gene.

\section{Acknowledgments}

The pathology review and meetings of the BCLC were supported by the European Union Concerted Action. On behalf of the BCLC, I would also like to thank all the families, clinicians and pathologists who have contributed to the studies. The pathology review group comprised Dr. S.R. Lakhani, Prof. J.P. Sloane, Prof. B.A. Gusterson, Dr. T. Anderson, Dr. J. Jacquemier, Dr. M.J. van de Vijver, Dr. L. Farid, Dr. D. Venter and Prof. M.R. Stratton. Dr. D. Easton and his team carried out the statistical analysis.

\section{References}

[1] Lakhani, S.R., Easton, D. and Stratton, M.R. on behalf of BCLC. Pathology of familial breast cancer: differences between breast cancers in carriers of BRCA1 or BRCA2 mutations and sporadic cases. Breast Cancer Linkage Consortium. Lancet 349, (1997) 1505-1510.

[2] Lakhani, S.R., Jacquemier, J., Sloane, J.P., Gusterson, B.A., Anderson, T.J., van de Vijver, M.J., Farid, L.M., Venter, D., Antoniou, A., Storfer-Isser, A., Smyth, E., Steel, C.M., Haites, N., Scott, R.J., Goldgar, D., Neuhausen, S., Daly, P.A., Ormiston, W., McManus, R., Scherneck, S., Ponder, B.A., Ford, D., Peto, J., Stoppa-Lyonnet, D., Easton, D.F. et al. Multifactorial analysis of differences between sporadic breast cancers and cancers involving BRCA1 and BRCA2 mutations. J. Nat. Cancer Inst. 90, (1998) 11381145 . 


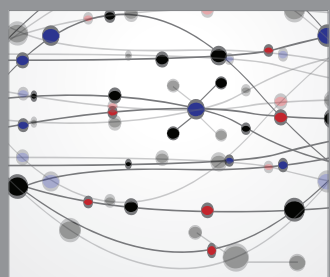

The Scientific World Journal
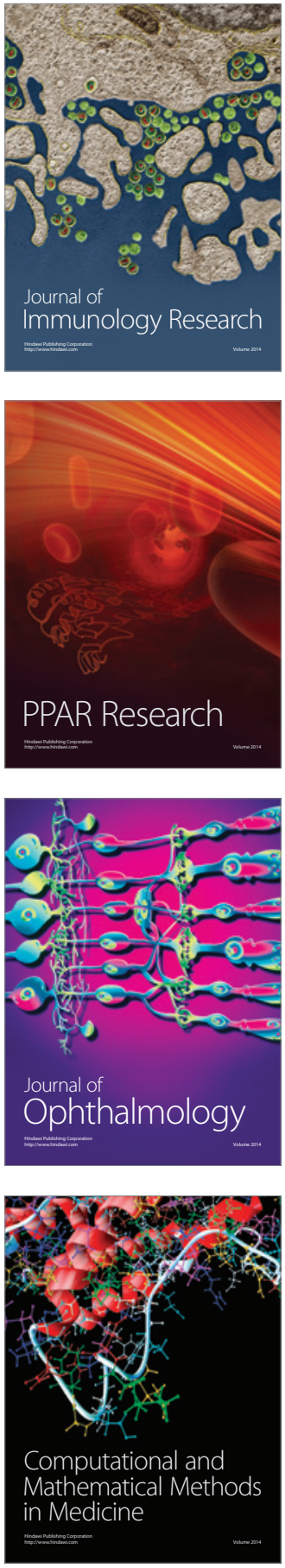

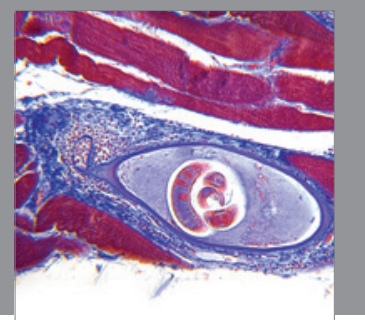

Gastroenterology

Research and Practice
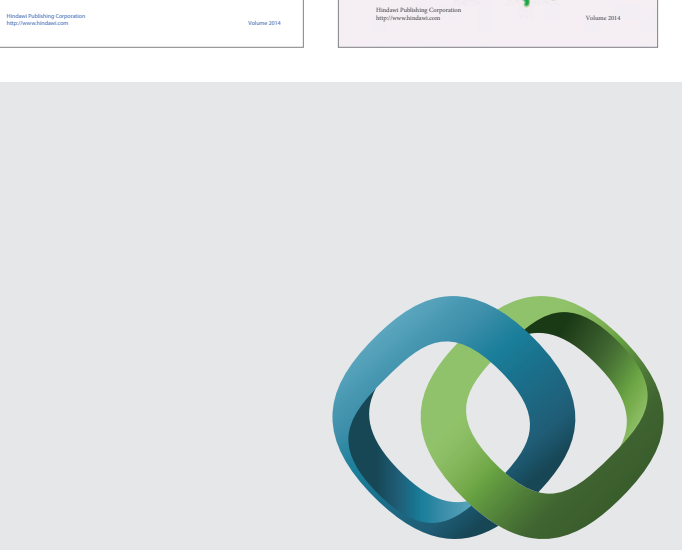

\section{Hindawi}

Submit your manuscripts at

http://www.hindawi.com
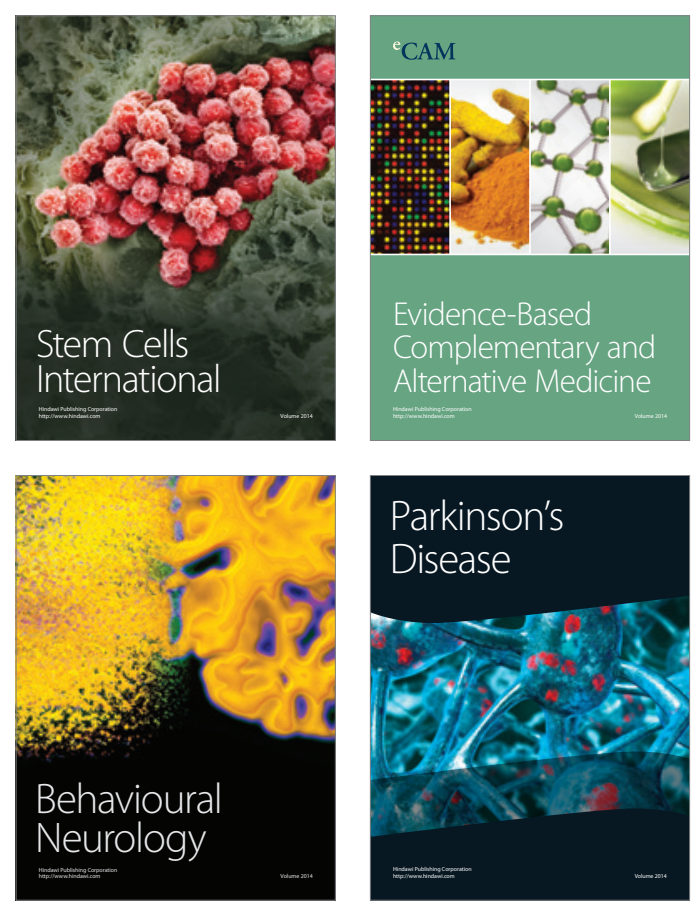

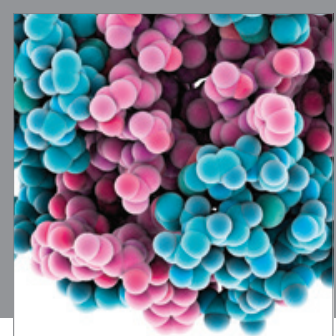

Journal of
Diabetes Research

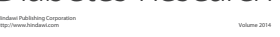

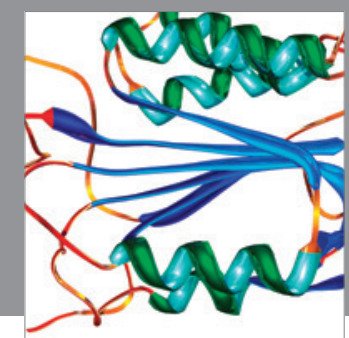

Disease Markers
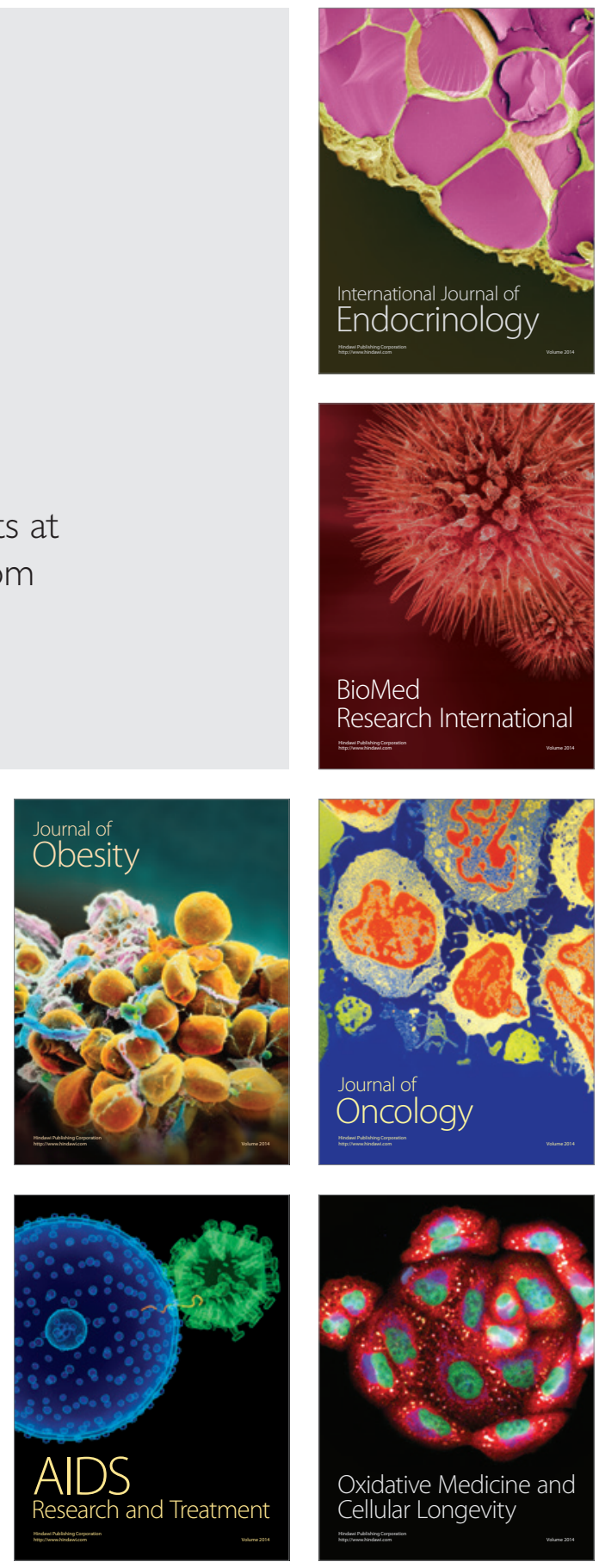